Research Article

\title{
Right $\mathrm{VI}$ palsy revealing nasopharyngeal cancer (UCNT)
}

\section{Abstract}

a. VI paralysis is a frequent and classic pathology in the clinical consultation of ophthalmology. The main difficulty is the diagnostic process (for the etiology).

b. In young adults, be aware of the possibility of a tumor with the correlation of rapid imaging.

c. UCNT is frequent in Morocco, its neurological symptoms are misleading because we do not always think of examining the nasopharynx properly in the event of damage to the cranial pairs.

d. Improving the prognosis for this cancer requires early diagnosis.

Keywords: nasopharynx, VI palsy, MRI
Volume 6 Issue 4 - 202 I

H Belatik, ${ }^{1,2}$ H Attifi, ${ }^{1,2}$ M Hmidi, ${ }^{1,2} \mathrm{~N}$

Touihem, ${ }^{1,2}$ A El boukhari, ${ }^{1,2}$ K Nadour ${ }^{1,2}$

'Department of otolaryngology, Moulay Ismail Military Hospital,

Meknes, Morocco

${ }^{2}$ Faculty of medicine and pharmacy, Sidi Mohamed Ben Abdellah

University, Fes, Morocco

Correspondence: $\mathrm{H}$ Belatik, department of otolaryngology, Moulay Ismail Military Hospital, Meknes, Morocco, 50000, faculty of medicine and pharmacy, Sidi Mohamed Ben Abdellah University, Fes, Morocco, 30000, Tel +2I 2664667962

Email belatikhamza@gmail.com

Received: August 06, 202I | Published: December 31, 202 I
Abbrevations: UCNT, Undifferentiated nasopharyngeal carcinomas; ENT, ear, nose, throat, and neck; MRI, Magnetic resonance imaging;

\section{Introduction}

VI palsy is characterized by damage to the lateral rectus muscle, preventing abduction of the paralyzed eye.

The aetiologies vary depending on the Age. In young adults, Any VI paralysis should primarily suggest a tumor cause.

Nasopharyngeal cancer is common in Morocco. Undifferentiated nasopharyngeal carcinomas (UCNT) constitute the predominant histological group.

Due to its deep basal cranial topography not very accessible to examination, and despite a rich but misleading borrowing symptomatology, this cancer remains a late diagnosis and a formidable prognosis.

The objective of our study is to encourage ENT surgeons to think about a UCNT in front of a paralysis of the VI.

\section{Material and methods}

This is a 46-year-old patient, with no particular pathological history, who presented for a consultation for an oculomotor disorder in his right eye with horizontal binocular diplopia (more marked in far vision) associated with occipital headaches in progressive installation (during 3 months) all evolving in a context of apyrexia.

\section{Results}

\section{The ophthalmological examination}

Shows a 10/10 VA (P2) in ERL with normal anterior and posterior segment.

oculomotor examination:

i. Compensatory attitude with the head turned to the right. ii. A constant esotropy of the right eye in the primary position (Figure 1).

iii. A deficit of abduction of the right eye (limitation of the external right ER and hyperaction of the internal right EL in favor of a paralysis of the VI of the right eye) (Figure 2).

iv. Horizontal binocular diplopia (red glass) which worsens in far vision and especially in the right lateral gaze.

v. The VI palsy of the right eye is confirmed by the Lancaster (Figure 3).

The general examination (ENT and neurological) and without particularities.

a) The ophthalmological examination is completed by a biological and radiological assessment and a neurological and ENT opinion.

Biology: The study of cerebrospinal fluid is normal.

EBV serology: high level of anti-EBV antibodies.

CT of the sinuses: nasal septum deviated to the left with thickening of the lower septa.

Orbito-cerebral MRI: in favor of a right nasopharyngeal neoplastic process, extended to the right cavernous compartment (Figure 4A,4B).

Histology: deep nasopharyngeal biopsy showing undifferentiated nasopharyngeal-type carcinoma (UCNT).

b) An alternating occlusion associated with analgesic treatment was started to limit the development of contractures and fight against pain.

\section{Discussion}

Among oculomotor palsies, VI (peripheral type) is the most common, around $44 \%$. It is often acquired and marked by a high percentage of recovery. ${ }^{1}$

A positive diagnosis is relatively straightforward, since this nerve invades only the ipsilateral lateral rectus. 


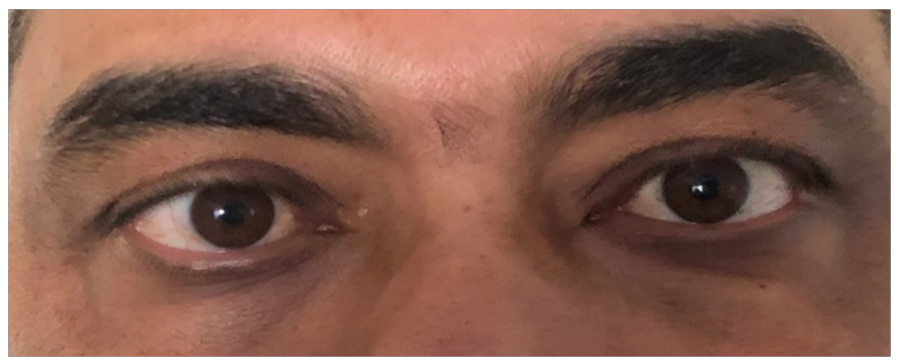

Figure I esotropia of the right eye in primary position.

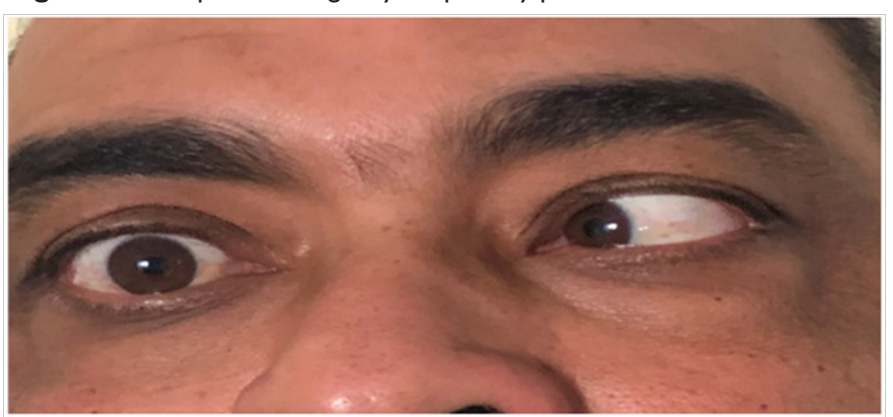

Figure 2 right eye abduction deficit.

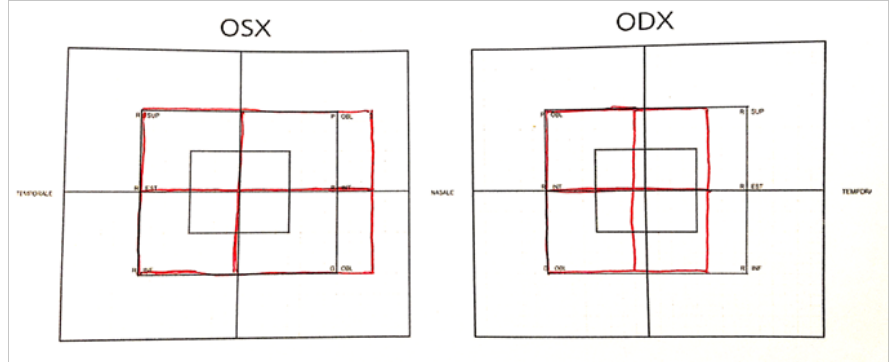

Figure 3 Lancaster in favor of $\mathrm{VI}$ palsy of the right eye.
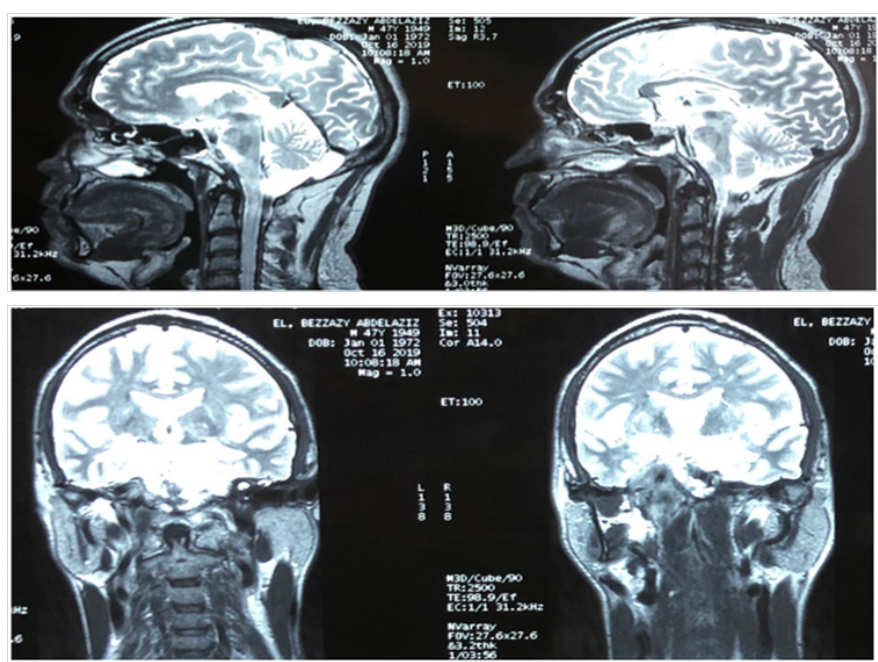

Figure 4A, 4B Orbito-cerebral MRI (coronal and sagittal slice) in favor of a right nasopharyngeal neoplastic process, extended to the right cavernous compartment.

The aetiologies vary depending on the age:

I. In older patients (average $65+/-3$ years), approximately $20 \%$ of VI paralysis is the consequence of microvascular disease. ${ }^{1}$
II. In children, Apart from congenital forms, the traumatic aetiology is most frequently found before neoplasias.

III. In the group of young adults (20-50 years): the tumor cause predominates ( $33 \%$ of cases).

In Morocco (intermediate risk areas), the incidence of nasopharyngeal cancer is high. It occurs at any age and the sex ratio is $3 \mathrm{H} / \mathrm{F}^{2}$

UNCT (the most frequent histological type) is a particular form due to its evolution, its high metastatic potential, its high radiosensitivity and its etiological relationship with EBV.

Cervical symptoms: lymphadenopathy (50 to $90 \%$ of cases) represent the most frequent circumstance of discovery, after otological $(25 \%)$ and rhinological (20\%) symptoms. ${ }^{3,4}$

Neurological symptoms (such as oculomotor paralysis of VI (diplopia), headaches, etc.) are the rarest (10 to $15 \%$ of cases); they are misleading and testify to an invasion of the base of the skull. ${ }^{5}$

$\mathrm{CT}$ and MRI are recognized as complementary imaging techniques for the initial assessment (locoregional extension). ${ }^{6,7}$

The dosage of anti-EBV antibodies is systematic before starting treatment. Their rate is often high in the case of UCNT. The antiVCA IgA level is very specific for undifferentiated nasopharyngeal carcinoma. $^{8}$

UCNT is a very chemo- and radiosensitive or even radiocurable tumor. Irradiation remains the standard treatment for this disease.?

The prognosis is poor, overall 5-year survival being 35\%. However, it is a function of the extension at the base of the skull that worsens the prognosis.

\section{Conclusion}

a) VI paralysis is a frequent and classic pathology in the clinical consultation of ophthalmology. The main difficulty is the diagnostic process (for the etiology).

b) In young adults, be aware of the possibility of a tumor with the correlation of rapid imaging.

c) UCNT is frequent in Morocco, its neurological symptoms are misleading because we do not always think of examining the nasopharynx properly in the event of damage to the cranial pairs.

d) Improving the prognosis for this cancer requires early diagnosis.

e) Early detection and screening are tested in endemic areas (antiEBV antibody/viral DNA).

\section{Acknowledgments}

None.

\section{Conflicts of interest}

The author states there are no conflicts of interest.

\section{Funding}

None.

\section{References}

1. Ed A, Péchereau J. Les paralysies oculomotrices. Nantes, Béatrice Roussat; Formes cliniques des Paralysies du Nervus Abducens (VI), p 53. Marie de Bideran; Épidémiologie des Paralysies Oculomotrices, 2007:5-6. 
2. Arfaoui A, Soulaymani A, Quyou A, et al. Le cancer du cavum au Maroc. Etude épidémiologique sur l'échantillon: Centre d'oncologie Al Azhar de Rabat. Antropo. 2007;14:75-82.

3. Li JX, Lu TX, Huang Y, et al. Clinical characteristics of recurrent nasopharyngeal carcinoma in high-incidence area. The Scientific World Journal. 2012.

4. Sham JST, Choy D, Wei WI, et al. Value of clinical follow-up for local nasopharyngeal carcinoma relapse. Head and Neck. 1992;14(3):208217.

5. Boussen H. Bouaouina N. Mokni - Baizig N. et al. Les carcinome du nasopharynx/données actuelles. Pathologie Biologie. 2004;11:15-22.
6. Sham JS, Cheung YK, Choy D, et al. Cranial nerve involvement and base of the skull erosion in nasopharyngeal carcinoma. Cancer. 1991;68(2):422-426.

7. Becker H. MRI findings delay the diagnosis of nasopharyngeal carcinoma. Clin Neuroradiol. 2011;21(3):153-156.

8. Altun M, Fandi A, Dupuis O, et al. Undifferentiated nasopharyngeal cancer (UCNT): current diagnostic and therapeutic aspects. Int J Radiat Oncol Biol Phys. 1995;32:859-877.

9. Kau HC, Tsai CC. New onset diplopia in patients with nasopharyngeal carcinoma following concurrent chemoradiotherapy: clinical features and etiology. BioMed Research International. 2015. 